\title{
Electrically Programmable Surfaces for Configurable Patterning of Cells**
}

\author{
By Chao Yung Fan, Yi-Chung Tung, Shuichi Takayama, Edgar Meyhöfer, * and \\ Katsuo Kurabayashi*
}

Microsystems technology provides the ability to precisely control micrometer-scale environments that surround cells in microfluidic devices, small-volume chambers, or biochips. ${ }^{[1]}$ Precise control over the chemical and cellular environment, including interactions with neighboring cells, in microdevices opens doors to new high-throughput and high-content experimental approaches and mechanistic insights into cell behaviors, such as relationships between cell shape and cell growth. ${ }^{[2]}$ Fundamental aspects of complex cell-cell interactions found in living organisms can also be re-created in microsystems for drug and toxicology screening. To create and accurately control the environmental and physiological conditions, surfaces capable of generating micrometer-scale patterns of cells in microsystems are crucial. These surfaces serve for controlling cellular growth factors, engineering tissues, and performing controlled cell-based assays, and could become key components for the development of bioelectronics and portable diagnostic devices useful in clinical settings. ${ }^{[1,3]}$

In this Communication, we present a new material approach that can be programmed to generate cell patterns on a surface, with the smallest dimension down to the size of single cells. The locations and shapes of the generated cell patterns on a surface can be controlled externally by selectively switching on microelectrode arrays in an engineered microfluidic device. In addition, the method allows one to configure different cell population densities and cell morphologies into generated cell patterns on the same surface by adjusting applied voltage biases on the microelectrodes.

Several techniques have demonstrated an ability to immobilize cells on designated regions on a surface. For example, cell patterns have been generated by using microcontact

[*] Prof. E. Meyhöfer, Prof. K. Kurabayashi Department of Mechanical Engineering University of Michigan

Ann Arbor, 48109-2125 MI (USA)

E-mail: meyhofer@umich.edu; katsuo@umich.edu

C. Y. Fan

Department of Electrical Engineering and Computer Science

University of Michigan

Ann Arbor, 48109-2125 MI (USA)

Y.-C. Tung, Prof. S. Takayama

Department of Biomedical Engineering

University of Michigan

Ann Arbor, 48109-2099 MI (USA)

[**] The authors thank Y.-W. Lin for assistance with electrode lithography. This work was supported by NSF, Yamatake Co., Japan, NIH, and an REA Fellowship from the University of Michigan (C.Y.F.). printing or lithography and surface modifications to define the location of cell attachments, ${ }^{[4-7]}$ microheaters to switch surface fouling properties through thermally responsive polymers, ${ }^{[3]}$ fluid flow to organize the location of cells in capillary networks, ${ }^{[8]}$ dielectrophoretic forces to concentrate cells into specific locations, ${ }^{[9]}$ ink-jet printing to place cells pixel by pixel on a substrate, ${ }^{[10]}$ and electrochemical reactions to cause surface chemistry changes or desorption of a self-assembled monolayer that leads to cell binding in specified regions. ${ }^{[4,11-14]}$ These techniques typically require the location of the cell patterns to be predetermined before the substrate for cell pattern construction is fabricated. Some techniques do allow cell adhesion on a surface to be switchable, but these can only achieve binary "yes" or "no" cell adhesion states. ${ }^{[4,11-13]}$ Other challenges associated with many past approaches include a need for open surfaces, which prohibits cell patterning in enclosed microfluidic channels, ${ }^{[10]}$ poor cell pattern definition resulting from thermal diffusion, ${ }^{[3]}$ and potential damages to cells owing to electrolysis and electrochemical reactions during the patterning process. ${ }^{[4,9,11-13]}$ The cell patterning technique that we have developed is fundamentally new and overcomes most of these shortcomings, based on the approach of controlling electrosurface phenomena with embedded microelectrodes. ${ }^{[15,16]}$ In this method, cells do not experience direct dc currents or electrolysis that might damage their structures.

In our previous study, ${ }^{[15]}$ we have achieved electrically programmable assembly of proteins, such as bovine serum albumen (BSA) and casein, on targeted regions on a hydrophobic dielectric surface. The surface was coated with a monolayer of Pluronic F108 (BASF) triblock copolymers, and localized protein adsorption was achieved by tuning the voltage bias on microelectrodes, which causes local variation in the steric repulsion of the triblock copolymers against proteins and achieves different degrees of protein surface adsorption. Inspired by the study, we herewith demonstrated programmable patterning of $\mathrm{C} 2 \mathrm{C} 12$ cells on an organic (Parylence C) dielectric surface in a microfluidic device (Fig. 1), which was constructed by using a simple one-mask fabrication process (see Experimental). The cell patterning demonstrated here also employs electrode biasing to cause a local electrowettingon-dielectric (EWOD) effect that leads to localized cell adhesion on the surface. ${ }^{[17]}$ But unlike the previous study, the cell patterning employs a low-surface-density layer of Pluronic triblock copolymers and a spatially uniform blanket layer of a cell-adhesive extracellular matrix (ECM) protein molecules (i.e., fibronectin). 


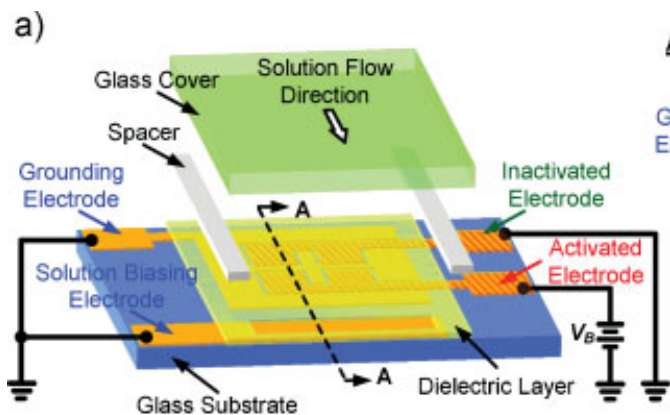

\section{A-A Cross-Section:}

b)

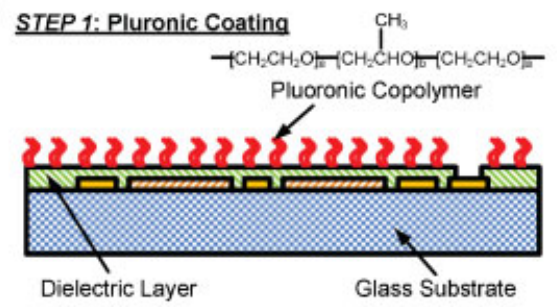

STEP 2: ECM Protein Coating

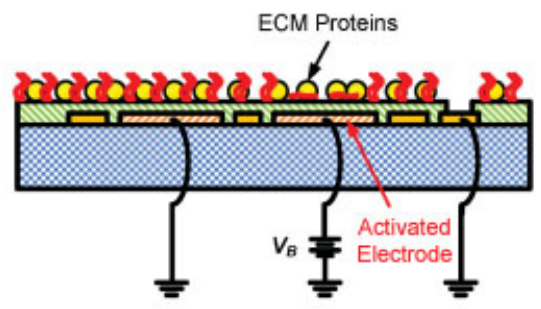

STEP 3: Cell Patterning
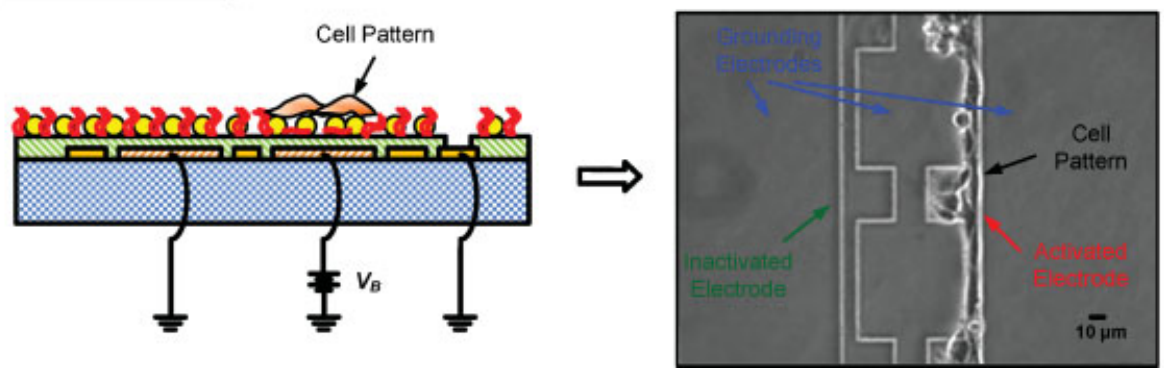

Figure 1. Schematics showing the cell patterning device design and procedure. a) $3 \mathrm{D}$ perspective view of the cell patterning device, and the corresponding cross-sectional view of the device substrate with microelectrodes and their electrical connections. The device chamber is about $2 \mathrm{~cm} \times 2 \mathrm{~cm} \times 200 \mu \mathrm{m}$ and is assembled by attaching the device piece containing microelectrode patterns to a microscope slide with ca. $200 \mu \mathrm{m}$ thick double-sided tape. b) Cell patterning procedure. Step 1: Pluronic copolymers are precoated on the surface. Step 2: Selected microelectrodes are activated and fibronectin proteins are perfused into the chamber to be adsorbed on the surface. Step 3: Cells are introduced into the chamber and incubated at $37{ }^{\circ} \mathrm{C}$ and $5 \% \mathrm{CO}_{2}$ for $1 \mathrm{~h}$ before unbound cells are washed away, revealing desired cell patterns on the surface (as shown in the micrograph obtained from an actual experiment). Following the washing process, the established cell patters remain restricted to the activated surfaces, even after the voltage bias has been removed.

In our method, the EWOD phenomenon occurs upon the application of voltage biases to the microelectrode arrays, causing the dielectric surface on the microelectrodes to wet (i.e., become more hydrophilic), rearranging copolymers on the region and switching the surface from a non-cell-adsorption state to a cell adsorption state. The initial non-adsorption state of the surface stems from the steric repulsion of the amphilic Pluronic copolymers, which have their hydrophobic poly(propylene oxide) (PPO) chains bound onto the hydrophobic dielectric device surface through the hydrophobichydrophobic interaction, leaving the copolymers' hydrophilic poly(ethylene oxide) PEO chains freely swaying in solution. ${ }^{[18]}$ In general, these PEO chains repel adsorbents from the surface, but as shown later, because of the low surface density of the Pluronic copolymers employed in our method, cell-adhesive fibronectin protein molecules are allowed to be adsorbed uniformly onto the surface while much larger sized cells are repelled from the surface by Pluronic's steric repulsion. We hypothesize that the switching of the surface to a cell adsorption state upon the application of bias voltages is mainly caused by either (i) the collapse of hydrophilic PEO copolymer chains onto the surface owing to the increased hydrophilichydrophilic interaction on the surface, or (ii) detachment of the copolymers from the reduced hydrophobic-hydrophobic interaction between the copolymers' hydrophobic PPO chains and the surface. ${ }^{[15,19]}$ Both cases suppress the steric repulsion exerted by the copolymers on cells and thus achieve spatially selective cell binding.

As the first step of the cell patterning process, a monolayer of Pluronic copolymers is initially formed on the device surface by flowing $100 \mu \mathrm{L}$ of a ca. $7.2 \mu \mathrm{g} \mathrm{mL} \mathrm{m}^{-1}$ Pluronic F108 solution into the device chamber (Step 1, Fig. 1b). The Pluronic copolymers are allowed to adsorb onto the surface for $10 \mathrm{~min}$. Then, bias voltages are applied to the selected microelectrode 
a)

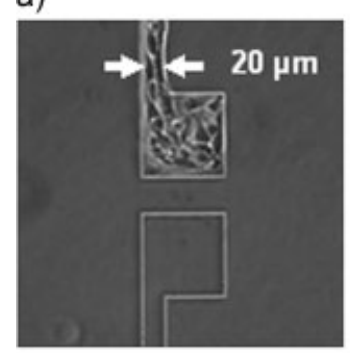

d)

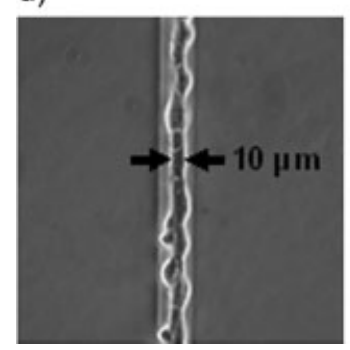

b)

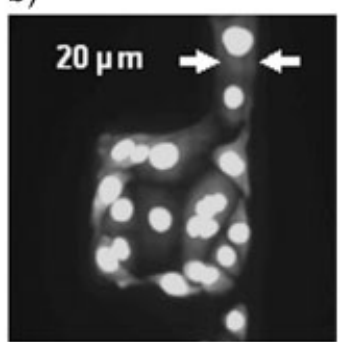

e)

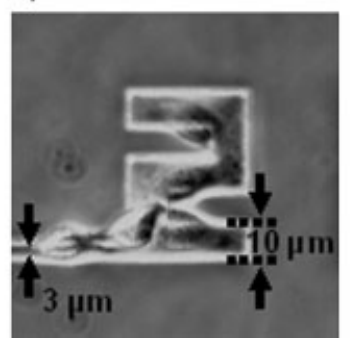

c)

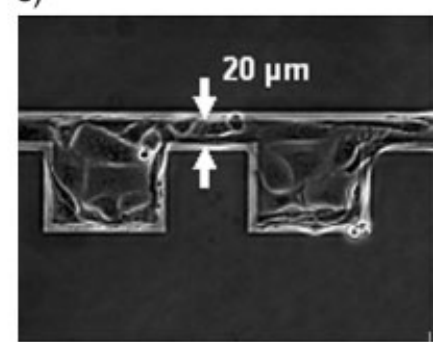

f)

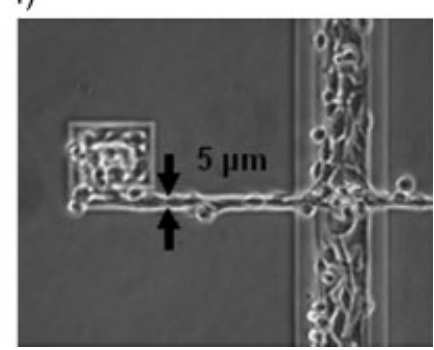

Figure 2. Programmable high-resolution cell patterning. a) Cells can be configured into different micropatterns by activating desired microelectrode patterns with a bias voltage (e.g., $60 \mathrm{~V}$ ), whereas microelectrodes that have no bias voltage applied $(0 \mathrm{~V})$ do not support cell attachment and growth. b) Fluorescence image of a cell pattern stained with Syto9, showing how cells adjust their shape to follow closely the contour of the microelectrode pattern. Cells can also be arranged into different pattern shapes, such as c) a sawtooth pattern, d) a line pattern with width down to single-cell dimensions, e) a number " 2 " pattern with width down to $3 \mu \mathrm{m}$, and f) a single-cell line pattern curled inward on a $5 \mu \mathrm{m}$-wide swirl-shaped microelectrode. Cell patterns are generated following the procedure shown in Figure 1 and are observed after $1 \mathrm{~h}$ incubation at $37^{\circ} \mathrm{C}$.

patterns and $1 \mathrm{~mL}$ deionized water is flown into the chamber to wash away unbound Pluronic copolymers. The device surface is subsequently coated with fibronectin by flowing $300 \mu \mathrm{L}$ of 10 to $60 \mu \mathrm{g} \mathrm{mL} \mathrm{m}^{-1}$ of fibronectin protein (F 2006, Sigma) diluted in deionized water into the device chamber (Step2, Fig. 1b). Fibronectin is allowed to bind to the surface for $30 \mathrm{~min}$ in a $37^{\circ} \mathrm{C}$ incubator before unbound protein molecules are washed away with $1 \mathrm{~mL}$ growth medium solution. To generate cell patterns, $300 \mu \mathrm{L}$ of $\mathrm{C} 2 \mathrm{C} 12$ myoblast cells in the growth medium solution are then flown into the chamber (Step 3, Fig. 1b). Cells are allowed to attach and spread on the device surface for ca. $1 \mathrm{~h}$ in a $37^{\circ} \mathrm{C}$ incubator at $5 \% \mathrm{CO}_{2}$. Afterward, unbound cells are washed away with $1 \mathrm{~mL}$ of the growth medium solution, electrical biases are removed from the microelectrodes, and then the patterned cells are observed under the microscope. In this process, the bias voltages were applied only during fibronectin coating and cell incubation for approximately $90 \mathrm{~min}$. The growth medium solution used in this procedure is composed of Dulbecco's Modified Eagle's Medium (DMEM; 11960; Gibco) containing 10\% v/v fetal bovine serum and $1 \% \mathrm{v} / \mathrm{v}$ antibiotic-antimycotic.

Following the cell patterning procedure described above, high-resolution cell patterns were successfully generated on the dielectric surface with the embedded microelectrodes of different shapes (Fig. 2). As shown by the optical micrographs in Figure 2, the presented cell patterning method has the ability to form cell patterns down to the single-cell dimension. The tion voltage of the device surface. ${ }^{[15]}$

patterned cells adjust their shapes to follow closely the contour of the activated microelectrode patterns. Moreover, the location of cell immobilization can be programmably determined by activating desired microelectrodes. We showed this by using two microelectrodes placed adjacent to each other (Fig. 2a). Cells only bound and spread onto the local surface over the selected microelectrode that was activated with a $60 \mathrm{~V}$ bias, while the other microelectrode remained inactivate with a $0 \mathrm{~V}$ bias.

Our method can also vary the surface density and morphology of the cells attached on the surface by adjusting the voltage magnitude applied to the microelectrode patterns (Fig. 3). Those cells that bind on the local surface with the microelectrode at $40 \mathrm{~V}$ are more rounded in shape, indicating that they are not spreading as much on the surface as compared to the case with $60 \mathrm{~V}$ applied bias. When the applied electrode voltage is decreased further, fewer cells are found to bind on the surface. The maximum operating voltage for this device has been fixed at $60 \mathrm{~V}$, the voltage approximately corresponding to the contact angle satura-

Using tetramethylrhodamine (TMR)-labeled fibronectin molecules and fluorescence microscopy, we observed that the voltage magnitude has negligible effects on the fibronectin surface density in this cell patterning method, in contrast to our previous voltage-controlled protein assembly process (Fig. 4). ${ }^{[15]}$ We found no noticeable variation in the fibronectin density across the entire device surface. This is attributed to the sparse Pluronic copolymers on the surface and indicates that changes in the surface adhesion behavior of the cells at varying electrode biases could result from the degree of the Pluronic copolymer rearrangement on the surface. According to the aforementioned hypotheses (i) and (ii), we believe that the increase in the wettability of the surface owing to the increase in the applied bias leads to either more copolymers to be released from the surface because of the reduced hydrophobic-hydrophobic interaction of the copolymers' hydrophobic PPO chains with the surface or more copolymers collapsing on the surface due to the increased hydrophilichydrophilic binding of the copolymers' PEO chains with the surface. As more copolymers collapse on or repel from the surface, cells would have more chance to contact the celladhesive fibronectin coating on the device surface. Therefore, increase in the applied voltage on electrodes enhances cell spreading and encourages more cells binding on the surface. It is also possible that the local electric field near the surface could affect the conformation of the fibronectin molecules, 
a)

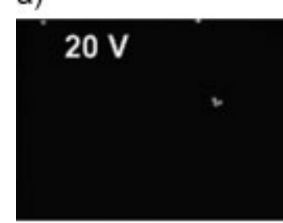

b)
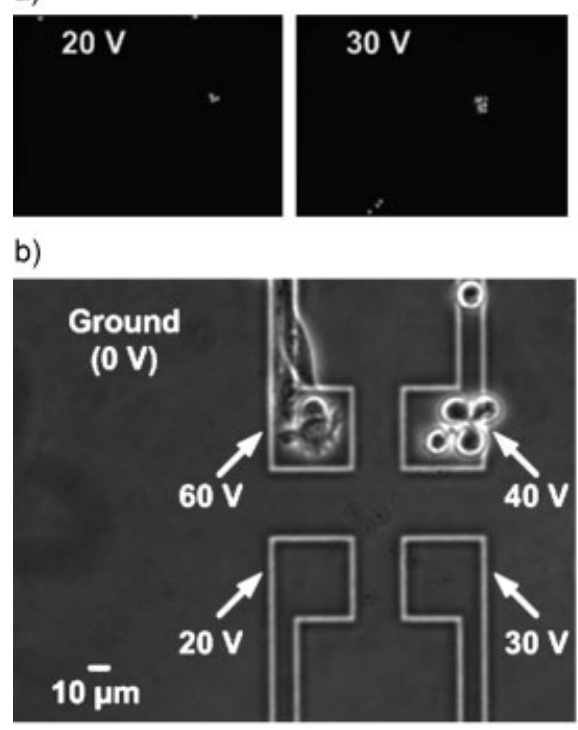
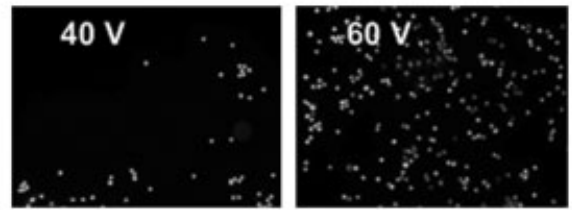

c)

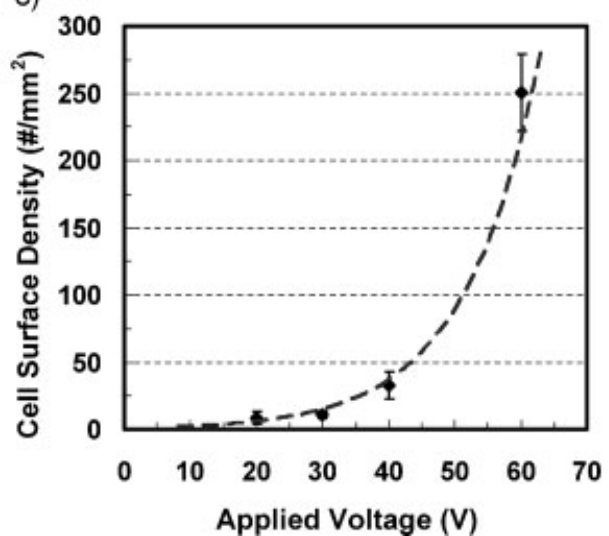

Figure 3. Cell surface density and cell morphology change with applied voltage magnitude. a) Number of cells increases with applied voltage, as shown by micrographs taken from regions on electrode pads (larger than the camera view) with different applied voltages $(20 \mathrm{~V}, 30 \mathrm{~V}, 40 \mathrm{~V}$, and $60 \mathrm{~V})$. All electrode pads are on the same device surface. Cells have been stained with Syto 9 fluorescence dye and observed under a fluorescence microscope using a $10 \times$ objective. b) Besides change in cell density, applied voltage magnitude also results in cell morphology changes. As shown on the four adjacent microelectrode patterns, cells spread much more on the local surface over the microelectrode with a higher applied bias (60 V) than the one with a lower applied bias (40 V). Patterns are imaged under brightfield using a $20 \times$ objective. c) Graph plotting how cell surface density increases exponentially with increase in the applied voltage bias. The data has been least-square fitted with an exponential equation. Each data point (mean $\pm \operatorname{standard~deviation,~} N=4$ ) is averaged over different electrode regions with the same applied voltage in the same device.

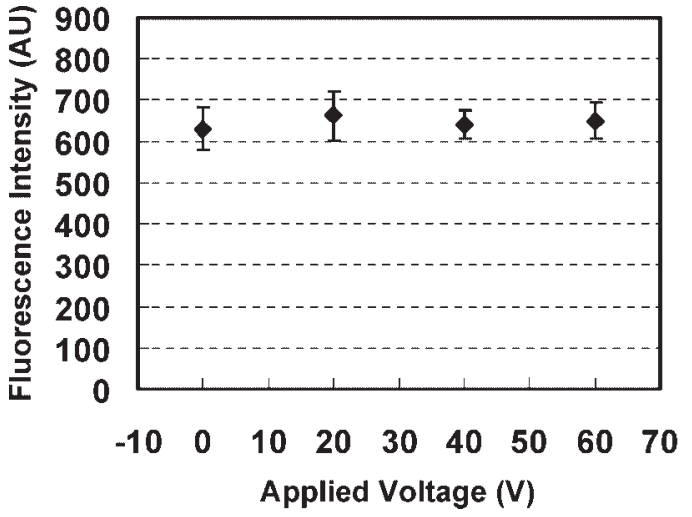

Figure 4. The effect of applied voltage magnitude on fibronectin protein surface density. Fibronectin proteins are labelled with TMR fluorescence dyes and observed under an inverted fluorescence microscope. As indicated by the similar fluorescence signals emitted from fibronectin proteins on surface locations over microelectrodes with different applied voltage biases, voltage magnitude has negligible effects on fibronectin surface density. Furthermore, a uniform and high fluorescence intensity was observed across the entire device surface. Because the Pluronic copolymer concentration (ca. $7.2 \mu \mathrm{g} \mathrm{mL}^{-1}$ ) used to coat the device surface is very low, Pluronic copolymers are expected to be sparsely distributed on the surface, allowing fibronectin proteins to be coated uniformly between Pluronic copolymers on the device surface. Therefore, we conclude that the selective cell binding above activated microelectrodes demonstrated here is not related to changes in fibronectin surface density, but is mainly due to the electrically programmed rearrangement of Pluronic copolymers on the local surface regions over activated microelectrodes. thus potentially contributing to the differences in the degree of the cell attachment. Studies are currently underway to fully understand the mechanisms that determine cell morphology and attachment on the activated surface regions.

The successful generation of cell patterns further provides evidence that fibronectin molecules are functional and are actively enhancing cell attachment on the surface, even in the presence of the biases applied to the microelectrode patterns. We have verified that the presence of fibronectin molecules was essential for the surface binding of the cells in the presented method (Fig. 5).

We have cultured patterned cells for a longer term to study their viability in the device chamber (Fig. 6). Here, we observed that patterned cells continued to spread and grow on top of the microelectrode. Cell growth was followed for up to 4.5 days, and during this entire observation period cells remained viable and were restricted to the microelectrode pattern, without the need to retain the voltage biases. This indicates that the pattering device has the ability to sustain the cell viability for long-term studies. Cell growth after removing the bias voltages has been observed to only happen on the surface region with the electrode that has previously been activated. The removal of the biases or the application of reverse biases does not release cells from the generated cell patterns. Therefore, the cell patterning process is irreversible. It suggests that only the Pluronic copolymers on activated regions are irreversibly repelled or reoriented on the surface by 
a) Cell Pattern With Fibronectin Coating

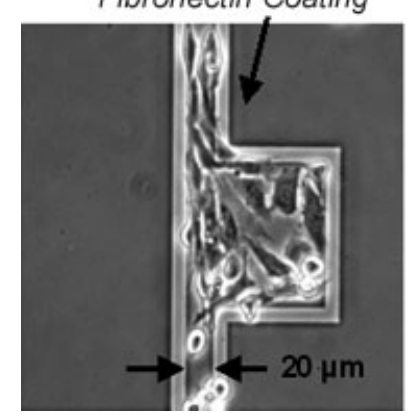

b) Cell Pattern Without Fibronectin Coating

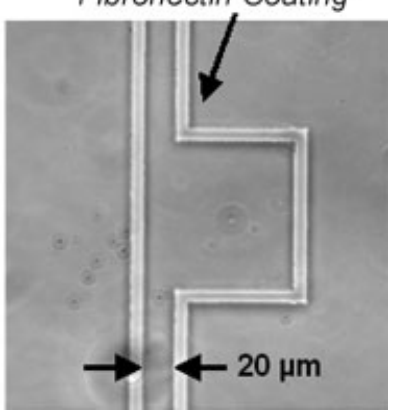

Figure 5. Fibronectin dependence of cell binding on local surfaces over activated microelectrode patterns. a) By using the same cell patterning procedure shown in Figure 1 that includes fibronectin coating the device surface before cells are introduced, cells are attached and spread well on regions over activated microelectrode patterns. b) On the other hand, when the same cell patterning procedure is repeated but without fibronectin coating, no cells bind to the surface even in the presence of activated microelectrodes. From the results we conclude that fibronectin coating is essential for generating cell patterns on the device surface; Furthermore, fibronectin molecules are active on the surface over activated microelectrodes as indicated by their ability to promote cell attachment on the surface. the local EWOD effect. Though, cells that are initially bound weakly on the surface tend to be detached from the surface after several hours.

In summary, an advanced material and methodology for cell patterning has been developed that allows one to program surfaces for the placement of cells. Within two hours, highdefinition cell patterns with flexible shapes and dimensions down to the single-cell level can be achieved. Furthermore, one can configure different cell population densities and cell morphologies on the generated cell patterns by adjusting the applied bias voltage on microelectrodes. The cell patterning method, with its ability to quickly program the placement of cell patterns on a microfluidic device surface without involving complex equipment or complicated surface treatments during the cell patterning procedure, could be valuable for developing portable, configurable bioelectronics and disposable devices for performing cell-based assays in cell-on-a-chip settings. Allowing for configuring different cell population densities and cell morphologies into generated cell micropatterns, the technique could enable future cell studies, such as unraveling the effect of cell morphology and cell population on cell-cell interactions and long-term cell growth. In addition, the ability to form high-definition flexibly shaped cell patterns is expected to significantly contribute to tissue engineering advances. Because cell shape can be confined by the contour of the microelectrodes, the technique may be extended to study a)

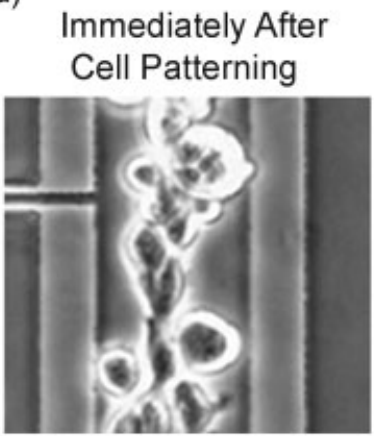

d)

After $\sim 2$ days

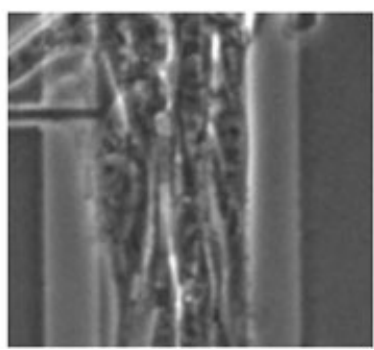

b)

After $\sim 6$ hours

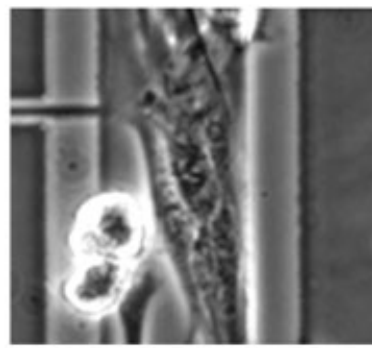

e) c)

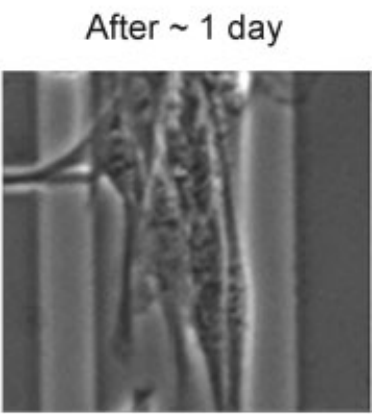

\section{After 4.5 days}

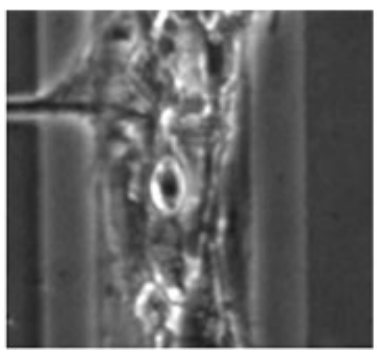

Figure 6. Long-term cell viability in device chamber. Here we present evidence that cells are viable in the device chamber for several days. The same cell patterning procedure shown in Figure 1 is used here to produce the cell pattern shown in (a). All electrical biases are then turned off and disconnected from the device at the end of the $1 \mathrm{~h}$ cell incubation. For long-term monitoring, the device is immersed in a $100 \mathrm{~mm}$ cell culture dish (Falcon) filled with a growth medium solution (DMEM with $10 \% \mathrm{v} / \mathrm{v}$ fetal bovine serum and $1 \% \mathrm{v} / \mathrm{v}$ antibiotic-antimycotic) and incubated at $37^{\circ} \mathrm{C}$ and $5 \% \mathrm{CO}_{2}$. The cell pattern is then monitored over time as shown by micrographs (b-e), which show the exact same surface location over time. Cells elongate and proliferate on the local surface above the microelectrode pattern over 4.5 days, an indication that they are viable during this time period. 
the effects of cell shapes on cell functions, motility, and migrations. ${ }^{[20]}$ We believe that the developed cell patterning technique, because of its voltage-controlled tuning of cell patterns and straightforward fabrication process, can be integrated into biological sensor and detector systems. The approach should also be useful to develop future microsystems for simulating cell-cell interactions and environments inside living organisms, as required for drug/toxicology screening and cancer research.

\section{Experimental}

Device Fabrication: Devices were made on ca. $150 \mu \mathrm{m}$ thick Corning glass wafers. Microelectrodes were fabricated via image reversal photolithography using PR5214 photoresist followed by a ca. $200 \mathrm{~nm}$ thick indium tin oxide (ITO) film deposition. Desired electrode patterns were formed by removing the sacrificial photoresist via a lift-off process. A ca. $0.8 \mu \mathrm{m}$ thick dielectric film of Parylene $\mathrm{C}$ was then coated over the entire device surface by chemical vapor deposition. The bonding pads for external wire connections and the "solution biasing electrode" were exposed by scratching away the Parylene C film with a razor blade.

Cell Staining and Fluorescence Microscopy: SYTO 9 fluorescence dye (Invitrogen) was used for the cell staining. After cells were patterned in the device, $300 \mu \mathrm{L}$ of a $5 \mu \mathrm{M}$ Syto 9 solution in DPBS was added to the device chamber. Approximately 5 min were allowed for cells to absorb the dye before fluorescence observation. An inverted fluorescence microscope (Nikon Eclipse TS100) with a $10 \times$ or $20 \times$ objective was used for fluorescence microscopy. Fluorescence signals were recorded using a digital CCD camera (CoolSnap cf2, Photometrics Inc.) and MetaVue (Version 7.0, Molecular Devices Co.) software.

Cell Culture Preparation: $\mathrm{C} 2 \mathrm{C} 12$ murine myoblasts were cultured in Dulbecco's Modified Eagle's Medium (DMEM; 11960; Gibco) with $20 \% \mathrm{v} / \mathrm{v}$ fetal bovine serum (FBS; 10082; Gibco), and $1 \% \mathrm{v} / \mathrm{v}$ antibiotic-antimicotic (15240; Gibco). The cultures were maintained on $100 \mathrm{~mm}$ cell culture dishes (Falcon) under $5 \% \mathrm{CO}_{2}$ and passaged by dissociation with $0.25 \%$ trypsin-EDTA (25200; Gibco). Cell suspensions for the experiments were made by dissociating cells from centrifugation at $1000 \mathrm{rpm}$ for $1 \mathrm{~min}$ at room temperature. An inverted routine microscope (Nikon Eclipse TS100) with either $10 \times$ or $20 \times$ objective was used for observing cell patterns. The images were recorded using a digital CCD camera (CoolSnap cf2, Photometrics Inc.) and MetaVue (Version 7.0, Molecular Devices Co.) software.

Measurement of Bias Effect on Fibronectin Protein Surface Density: Fibronection surface density data in Figure 4 were obtained by closely following the cell patterning procedure shown in Figure 1b: Initially, a monolayer of Pluronic copolymers was adsorbed on the device surface by flowing $100 \mu \mathrm{L}$ of ca. $7.2 \mu \mathrm{g} \mathrm{mL}^{-1}$ Pluronic F108 solution into the device chamber and incubating for $10 \mathrm{~min}$ while applying bias voltages to the microelectrodes. Unbound Pluronic copolymers were washed away with $1 \mathrm{~mL}$ deionized water. To coat fibronectin proteins on the device surface, $300 \mu \mathrm{L}$ of $10 \mu \mathrm{g} \mathrm{mL} \mathrm{m}^{-1}$ of TMR-labelled fibronectin diluted in deionized water was then introduced into the device chamber. Fibronectin molecules were allowed to adsorb to the surface for $30 \mathrm{~min}$ before unbound protein molecules were washed away with $1 \mathrm{~mL}$ de-onized water. TMR-labelled fibronectin molecules on the surface were observed by inverted fluorescence microscopy with $40 \times$ oil-immersion objective, and fluorescence signals were recorded with a digital CCD camera and quantified off-line using Image $\mathrm{J}$.

Received: August 29, 2007

Revised: October 9, 2007

Published online: March 20, 2008

[1] J. El-Ali, P. K. Sorger, K. F. Jensen, Nature 2006, 442, 403.

[2] C. S. Chen, M. Mrksich, S. Huang, G. M. Whitesides, D. E. Ingber, Science 1997, 276, 1425.

[3] X. Cheng, Y. Wang, Y. Hanein, K. F. Bohringer, B. D. Ratner, J. Biomed. Mater. Res. 2004, 70A, 159.

[4] X. Jiang, R. Ferrigno, M. Mrksich, G. M. Whitesides, J. Am. Chem. Soc. 2003, 125, 2366.

[5] D. I. Rozkiewicz, Y. Kraan, M. W. Werten, F. A. de Wolf, V. Subramaniam, B. J. Ravoo, D. N. Reinhoudt, Chem. Eur. J. 2006, 12,6290 .

[6] S. Bouaidat, C. Berendsen, P. Thomsen, S. G. Petersen, A. Wolff, J. Jonsmann, Lab Chip 2004, 4, 632.

[7] I. S. Carrico, S. A. Maskarinec, S. C. Heilshorn, M. L. Mock, J. C. Liu, P. J. Nowatzki, C. Franck, G. Ravichandran, D. A. Tirrell, J. Am. Chem. Soc. 2007, 129, 4874.

[8] E. Delamarche, A. Bernard, H. Schmid, B. Michel, H. Biebuyck, Science 1997, 276, 779.

[9] D. R. Albrecht, G. H. Underhill, T. B. Wassermann, R. L. Sah, S. N. Bhatia, Nat. Methods 2006, 3, 369.

[10] E. A. Roth, T. Xu, M. Das, C. Gregory, J. J. Hickman, T. Boland, Biomaterials 2004, 25, 3707.

[11] H. Kaji, M. Kanada, D. Oyamatsu, T. Matsue, M. Nishizawa, Langmuir 2004, 20, 16.

[12] Y. Li, B. Yuan, H. Ji, D. Han, S. Chen, F. Tian, X. Jiang, Angew. Chem. Int. Ed. 2007, 46, 1094.

[13] W.-S. Yeo, M. Mrksich, Adv. Mater. 2004, 16, 1352.

[14] X. Zhu, K. L. Mills, P. R. Peters, J. H. Bahng, E. H. Liu, J. Shim, K. Naruse, M. E. Csete, M. D. Thouless, S. Takayama, Nat. Mater. 2005, 4, 403.

[15] C. Y. Fan, K. Kurabayashi, E. Meyhofer, Nano Lett. 2006, 6, 2763.

[16] J. Lahann, S. Mitragotri, T.-N. Tran, H. Kaido, J. Sundaram, I. S. Choi, S. Hoffer, G. A. Somorjai, R. Langer, Science 2003, 299, 371.

[17] F. Mugele, J.-C. Baret, J. Phys. Condens. Matter 2005, 17, R705.

[18] T. Arnebrant, M. C. Wahlgren, in Proteins at Interfaces II: Fundamentals and Applications (Eds: T. A. Horbett, J. L. Brash), ACS, Washington, DC 1995.

[19] J. D. Andrade, V. Hlady, A. P. Wei, Pure Appl. Chem. 1992, 64, 1777.

[20] X. Jiang, D. A. Bruzewicz, A. P. Wong, M. Piel, G. M. Whitesides, Proc. Natl. Acad. Sci. USA 2005, 102, 975. 